PROCEEDINGS OF THE

AMERICAN MATHEMATICAL SOCIETY

Volume 132, Number 12, Pages 3629-3639

S 0002-9939(04)07624-5

Article electronically published on July 20, 2004

\title{
STRONG UNIQUENESS FOR THE PLATE EQUATIONS
}

\author{
SHIGEO TARAMA
}

(Communicated by David S. Tartakoff)

\begin{abstract}
In this paper we show the strong uniqueness for the plate equations. By using the idea due to Lebeau we transform the given operator to the elliptic operators to which we apply the Carleman estimates given by Alinhac and Lerner.
\end{abstract}

\section{INTRODUCTION}

The strong uniqueness for operators whose order is greater than 2 is not well studied except for the iterated Laplace operators (see, for example, Colombini and Grammatico 22 and the references therein). In this paper we consider the strong uniqueness for the plate equations.

Let $U$ be a neighborhood of $\{0\} \times(-\delta, \delta)$, with some $\delta>0$, in $\mathbb{R}_{x}^{n} \times \mathbb{R}_{t}$, and let $P\left(x, \partial_{x}, D_{t}\right)$ be a differential operator with coefficients depending only on the variables $x$ given by

$$
P\left(x, \partial_{x}, D_{t}\right)=\rho(x) D_{t}^{2}-\Delta_{x}^{2}+a\left(x, \partial_{x}\right) \Delta_{x}+b\left(x, \partial_{x}\right)
$$

where $\rho(x)$ is a positive smooth function and $a\left(x, \partial_{x}\right)$ and $b\left(x, \partial_{x}\right)$ differential operators with smooth coefficients of orders 1 and 2, respectively. Here we use the notation $D_{t}=\frac{1}{i} \partial_{t}$ and $\Delta_{x}=\sum_{1 \leq j \leq n} \partial_{x_{j}}^{2}$.

We say that a function $u(x, t)$ on a neighborhood of $\{0\} \times(-\delta, \delta)$ in $\mathbb{R}_{x}^{n} \times \mathbb{R}_{t}$ is flat on $\{0\} \times(-\delta, \delta)$ when $u(x, t)$ and its derivatives of any order vanish on $\{0\} \times(-\delta, \delta)$. We show in this paper the following theorem.

Theorem 1.1. Let $u(x, t)$ be a smooth function that is flat on $\{0\} \times(-\delta, \delta)$ and satisfies

$$
P u=0
$$

on a neighborhood of $\{0\} \times(-\delta, \delta)$ in $\mathbb{R}_{x}^{n} \times \mathbb{R}_{t}$. Then $u(x, t)$ is identically zero on some neighborhood of $\{0\} \times\{0\}$.

The plan of the proof is the following. First using the idea of Lebeau [4] (see also Robbiano [5] and Tataru [6]) we transform the given operator $P$ to the product of second-order elliptic operators. Then, applying the Carleman estimates given by Alinhac and Lerner [1] to our transformed operator, we draw the conclusion of Theorem [1.1 Since the Carleman estimates due to Alinhac and Lerner [1] are crucial for our argument, we give the outline of its proof in the appendix.

Received by the editors August 21, 2003.

2000 Mathematics Subject Classification. Primary 35A07, 35Q72.

Key words and phrases. Strong uniqueness, plate equations.

(C)2004 American Mathematical Society 
We remark that in our reasoning it is essential that the coefficients are time independent, and we remark also that for plate equations and related equations there are many works on the unique continuation of solutions (see, for example, Isakov [3]).

In the following, we denote by $C^{\infty}(\Omega)$ the space of all infinitely differentiable functions on $\Omega$ and by $C_{0}^{\infty}(\Omega)$ the space that consists of compactly supported functions belonging to $C^{\infty}(\Omega)$. For $f(x, t)$ and $g(x, t)$, we use the notation $(f, g)$ and $\|f\|$ defined by

$$
\begin{gathered}
(f, g)=\int f(x, t) \overline{g(x, t)} d x d t, \\
\|f\|=\sqrt{(f, f)} .
\end{gathered}
$$

We also use the standard notation of multi-indices. The constants appearing in the formulas may be different line by line.

\section{Proof of Theorem 1.1}

We assume that all the differential operators appearing in this section have smooth coefficients. Let $\phi(t)$ be a function in $\gamma_{0}{ }^{(\alpha)}((-\delta, \delta))$ with $1<\alpha<2$ and $\delta>0$, that is to say, $\phi(t) \in C_{0}{ }^{\infty}((-\delta, \delta))$ and there exist positive constants $K_{1}$ and $K_{2}$ such that for any integer $k \geq 0$ we have

$$
\left|\phi^{(k)}(t)\right| \leq K_{1} K_{2}{ }^{k}(k !)^{\alpha} \text { for any } t \in(-\delta, \delta) .
$$

Let

$$
\begin{aligned}
\Psi(z) & =\cosh (\sqrt{z}) \\
& =\sum_{n=0}^{\infty} \frac{z^{n}}{(2 n) !} .
\end{aligned}
$$

Now we define the operator $T_{\phi}$ by

$$
\begin{aligned}
T_{\phi}(v) & =\left\langle\Psi\left(\lambda^{2} D_{t}\right) v(t), \phi(t)\right\rangle \\
& =\left\langle v(t), \Psi\left(-\lambda^{2} D_{t}\right) \phi(t)\right\rangle \\
& =\frac{1}{2 \pi} \int_{\mathbb{R}^{3}} e^{i(s-t) \tau} v(s) \Psi\left(-\lambda^{2} \tau\right) \phi(t) d t d \tau d s .
\end{aligned}
$$

Since we see from (2.1) that the Fourier transform of $\phi(t)$ that is denoted by $\hat{\phi}(\tau)$ satisfies

$$
|\hat{\phi}(\tau)| \leq C_{1} e^{-C_{2}|\tau| \frac{1}{\alpha}}
$$

with positive constants $C_{1}$ and $C_{2}$, then we see from

$$
\left|\Psi\left(-\lambda^{2} \tau\right)\right| \leq e^{|\lambda||\tau|^{\frac{1}{2}}}
$$

that the integral (2.2) converges for any $v(t) \in C_{0}^{\infty}(\mathbb{R})$. Furthermore since

$$
\Psi\left(-\lambda^{2} D_{t}\right)=\sum_{n=0}^{\infty} \frac{\lambda^{2 n}}{(2 n) !}\left(-D_{t}\right)^{n}
$$


and $\phi(t) \in \gamma_{0}{ }^{(\alpha)}((-\delta, \delta))$ with $1<\alpha<2$, we see that

$$
\Psi\left(-\lambda^{2} D_{t}\right) \phi(t)=\sum_{n=0}^{\infty} \frac{\lambda^{2 n}}{(2 n) !}\left(-D_{t}\right)^{n} \phi(t)
$$

is an entire function of $\lambda$ with values in $C_{0}^{\infty}((-\delta, \delta))$. Then $T_{\phi}(v)$ is well-defined for any $v \in C^{\infty}((-\delta, \delta))$. Since it follows from $\Psi\left(\lambda^{2} D_{t}\right)=\cosh \left(\lambda \sqrt{D_{t}}\right)$ that

$$
\partial_{\lambda}^{2} \Psi\left(\lambda^{2} D_{t}\right)=D_{t} \Psi\left(\lambda^{2} D_{t}\right)
$$

then we see that

We remark that

$$
\partial_{\lambda}^{2} T_{\phi}(v)=T_{\phi}\left(D_{t} v\right)
$$

$$
\left.T_{\phi}(v)\right|_{\lambda=0}=\int_{\mathbb{R}} \phi(t) v(t) d t .
$$

Let $u(x, t) \in C^{\infty}\left(\left\{(x, t) \in \mathbb{R}_{x}^{n} \times \mathbb{R}_{t}|| x \mid<r_{1}\right.\right.$ and $\left.\left.|t|<\delta\right\}\right)$ with $r_{1}, \delta>0$ flat on $\{0\} \times(-\delta, \delta)$ and satisfying $P u=0$ on $\left\{(x, t) \in \mathbb{R}_{x}^{n} \times \mathbb{R}_{t}|| x \mid<r_{1}\right.$ and $\left.|t|<\delta\right\}$. We define $U(x, \lambda)$ by

$$
U(x, \lambda)=T_{\phi}(u(x, \cdot))
$$

Then $U(x, \lambda)$ is an entire function of $\lambda$ with values in $C^{\infty}\left(\left\{x \in \mathbb{R}_{x}^{n}|| x \mid<r_{1}\right\}\right)$ and satisfies

$$
\rho(x) \partial_{\lambda}^{4} U-\Delta_{x}^{2} U+a\left(x, \partial_{x}\right) \Delta_{x} U+b\left(x, \partial_{x}\right) U=0 .
$$

Since $\rho(x)>0$, we see that for a first-order differential operator $A\left(x, \partial_{x}\right)$,

$$
\begin{aligned}
& \left(\rho^{\frac{1}{2}}(x) \partial_{\lambda}^{2}+\Delta_{x}-A\left(x, \partial_{x}\right)\right)\left(\rho^{\frac{1}{2}}(x) \partial_{\lambda}^{2}-\Delta_{x}+A\left(x, \partial_{x}\right)\right) \\
& \quad=\rho(x) \partial_{\lambda}^{4}-\Delta_{x}^{2}+\left(2 A\left(x, \partial_{x}\right)+\left[\Delta_{x}, \rho^{\frac{1}{2}}(x)\right] \rho^{-\frac{1}{2}}(x)\right) \Delta_{x} \\
& \quad+\left[\Delta_{x}-A\left(x, \partial_{x}\right), \rho^{\frac{1}{2}}(x)\right] \rho^{-\frac{1}{2}}(x)\left(\rho^{\frac{1}{2}}(x) \partial_{\lambda}^{2}-\Delta_{x}+A\left(x, \partial_{x}\right)\right)+C\left(x, \partial_{x}\right)
\end{aligned}
$$

with a second-order differential operator $C\left(x, \partial_{x}\right)$.

Hence by picking

$$
A\left(x, \partial_{x}\right)=\frac{1}{2}\left(a\left(x, \partial_{x}\right)-\left[\Delta_{x}, \rho^{\frac{1}{2}}(x)\right] \rho^{-\frac{1}{2}}(x)\right)
$$

and by setting

$$
V(x, \lambda)=\rho^{\frac{1}{2}}(x) \partial_{\lambda}^{2} U(x, \lambda)-\left(\Delta_{x}-A\left(x, \partial_{x}\right)\right) U(x, \lambda),
$$

we see from (2.5) and (2.6) that $U(x, \lambda)$ and $V(x, \lambda)$ satisfy the system of equations

$$
\begin{aligned}
& \rho^{\frac{1}{2}}(x) \partial_{\lambda}^{2} U-\left(\Delta_{x}-A\left(x, \partial_{x}\right)\right) U=V \\
& \rho^{\frac{1}{2}}(x) \partial_{\lambda}^{2} V+\left(\Delta_{x}-A\left(x, \partial_{x}\right)\right) V=B_{1}\left(x, \partial_{x}\right) V+B_{2}\left(x, \partial_{x}\right) U
\end{aligned}
$$

where $B_{j}\left(x, \partial_{x}\right)$ is a differential operator of order $j$.

Since $U(x, \lambda)$ and $V(x, \lambda)$ are entire functions of $\lambda$, we get

$$
\begin{gathered}
\partial_{\lambda}^{2} U\left(x, t_{1}+i t_{2}\right)=-\partial_{t_{2}}^{2} U\left(x, t_{1}+i t_{2}\right)=-\partial_{t_{1}}^{2} U\left(x, t_{1}+i t_{2}\right)-2 \partial_{t_{2}}^{2} U\left(x, t_{1}+i t_{2}\right), \\
\partial_{\lambda}^{2} V\left(x, t_{1}+i t_{2}\right)=\partial_{t_{1}}^{2} V\left(x, t_{1}+i t_{2}\right)=2 \partial_{t_{1}}^{2} V\left(x, t_{1}+i t_{2}\right)+\partial_{t_{2}}^{2} V\left(x, t_{1}+i t_{2}\right) .
\end{gathered}
$$

Therefore, by putting

$$
\begin{aligned}
& W_{1}\left(x, t_{1}, t_{2}\right)=U\left(x, t_{1}+i t_{2}\right), \\
& W_{2}\left(x, t_{1}, t_{2}\right)=V\left(x, t_{1}+i t_{2}\right),
\end{aligned}
$$


we see that $W_{1}\left(x, t_{1}, t_{2}\right)$ and $W_{2}\left(x, t_{1}, t_{2}\right)$ satisfy the following elliptic system:

$$
\begin{aligned}
\rho^{\frac{1}{2}}(x) \partial_{t_{1}}^{2} W_{1}+2 \rho^{\frac{1}{2}}(x) \partial_{t_{2}}^{2} W_{1}+\left(\Delta_{x}-A\left(x, \partial_{x}\right)\right) W_{1}= & -W_{2}, \\
2 \rho^{\frac{1}{2}}(x) \partial_{t_{1}}^{2} W_{2}+\rho^{\frac{1}{2}}(x) \partial_{t_{2}}^{2} W_{2}+\left(\Delta_{x}-A\left(x, \partial_{x}\right)\right) W_{2}= & B_{1}\left(x, \partial_{x}\right) W_{2} \\
& +B_{2}\left(x, \partial_{x}\right) W_{1}
\end{aligned}
$$

on $\left\{\left(x, t_{1}, t_{2}\right) \in \mathbb{R}_{x}^{n} \times \mathbb{R}_{t}^{2}|| x \mid<r_{1}\right\}$.

Since $u(x, t)$ is flat on $\{0\} \times(-\delta, \delta), U(x, \lambda)$ is flat on $\{0\} \times \mathbb{C}$. Then $W_{1}\left(x, t_{1}, t_{2}\right)$ and $W_{2}\left(x, t_{1}, t_{2}\right)$ are flat on $\{0\} \times \mathbb{R}_{t}^{2}$.

Now we apply the Carleman estimates due to Alinhac and Lerner [1] to our system. First of all we make the change of coordinates

$$
x=\left(1-|T|^{2}\right) X, \quad t=T .
$$

Then we see that

and

$$
\left(1-|t|^{2}\right) \frac{\partial}{\partial x_{j}}=\frac{\partial}{\partial X_{j}}
$$

$$
\left(1-|t|^{2}\right) \frac{\partial}{\partial t_{k}}=\left(1-|T|^{2}\right) \frac{\partial}{\partial T_{k}}+2 T_{k} \sum_{j=1}^{n} X_{j} \frac{\partial}{\partial X_{j}} .
$$

Hence we see that $w_{1}(X, T)$ and $w_{2}(X, T)$, given by

$$
\begin{aligned}
& w_{1}(X, T)= \begin{cases}W_{1}\left(\left(1-|T|^{2}\right) X, T\right) & |T| \leq 1, \\
0 & |T| \geq 1,\end{cases} \\
& w_{2}(X, T)= \begin{cases}W_{2}\left(\left(1-|T|^{2}\right) X, T\right) & |T| \leq 1, \\
0 & |T| \geq 1,\end{cases}
\end{aligned}
$$

satisfy

$$
\begin{aligned}
\rho^{\frac{1}{2}}(0)\left(1-|T|^{2}\right)^{2}\left(\partial_{T_{1}}^{2}+\right. & \left.2 \partial_{T_{2}}^{2}\right) w_{1} \\
& +\Delta_{X} w_{1}+A_{1}\left(X, T, \partial_{X}, \partial_{T}\right) w_{1}=-\left(1-|T|^{2}\right)^{2} w_{2}, \\
\rho^{\frac{1}{2}}(0)\left(1-|T|^{2}\right)^{2}\left(2 \partial_{T_{1}}^{2}+\partial_{T_{2}}^{2}\right) & w_{2} \\
+\Delta_{X} w_{2} & +A_{2}\left(X, T, \partial_{X}, \partial_{T}\right) w_{2} \\
& =C_{1}\left(X, T, \partial_{X}, \partial_{T}\right) w_{2}+C_{2}\left(X, T, \partial_{X}, \partial_{T}\right) w_{1}
\end{aligned}
$$

where $A_{j}\left(X, T, \partial_{X}, \partial_{T}\right)$ and $C_{j}\left(X, T, \partial_{X}, \partial_{T}\right)$ are differential operators given by

$$
A_{j}\left(X, T, \partial_{X}, \partial_{T}\right)=\sum_{|\alpha|+|\beta| \leq 2} a_{j, \alpha, \beta}(X, T)\left(1-|T|^{2}\right)^{|\beta|} \partial_{x}^{\alpha} \partial_{T}^{\beta}
$$

with the coefficients $a_{j, \alpha, \beta}(X, T)$ that satisfy $a_{j, \alpha, \beta}(0, T)=0$ if $|\alpha|+|\beta|=2$, and

$$
C_{j}\left(X, T, \partial_{X}, \partial_{T}\right)=\sum_{|\alpha|+|\beta| \leq j} c_{j, \alpha, \beta}(X, T)\left(1-|T|^{2}\right)^{|\beta|} \partial_{x}^{\alpha} \partial_{T}^{\beta} .
$$

We denote by $Q_{1}$ and $Q_{2}$ the operators appearing in (2.9) and (2.10):

$$
\begin{aligned}
& Q_{1}=\rho^{\frac{1}{2}}(0)\left(1-|T|^{2}\right)^{2}\left(\partial_{T_{1}}^{2}+2 \partial_{T_{2}}^{2}\right)+\Delta_{X}+A_{1}\left(X, T, \partial_{X}, \partial_{T}\right), \\
& Q_{2}=\rho^{\frac{1}{2}}(0)\left(1-|T|^{2}\right)^{2}\left(2 \partial_{T_{1}}^{2}+\partial_{T_{2}}^{2}\right)+\Delta_{X}+A_{2}\left(X, T, \partial_{X}, \partial_{T}\right) .
\end{aligned}
$$

Recall the Carleman estimates due to Alinhac-Lerner [1] for $Q_{1}$ and $Q_{2}$. 
Lemma 2.1 (Alinhac-Lerner [1]). There exist positive constants $r_{0} \in(0,1 / 10)$ and $\gamma_{0}>0$ such that we have for any $w(X, T) \in C^{\infty}\left(\mathbb{R}_{X}^{n} \times \mathbb{R}_{T}^{2}\right)$ supported in $\left\{(X, T) \in \mathbb{R}_{X}^{n} \times \mathbb{R}_{T}^{2}|| X \mid \leq r_{0}\right.$ and $\left.|T| \leq 1\right\}$ and flat on $X=0$,

$$
\begin{aligned}
\gamma^{-1}\left(\sum_{k+|\alpha|+|\beta|=2} \gamma^{2 k}\left\|\Phi^{-\gamma}|\log r|^{-1} r^{|\alpha|+|\beta|}\left(1-|T|^{2}\right)^{|\beta|} \partial_{X}^{\alpha} \partial_{T}^{\beta} w\right\|^{2}\right) & \\
& \leq C\left\|r^{2} \Phi^{-\gamma} Q_{j} w\right\|^{2}
\end{aligned}
$$

for $\gamma>\gamma_{0}$ and $j=1,2$, where $r=|X|$ and $\Phi=|\log r| r$.

We give a sketch of the proof of the estimates above in the appendix.

Taking a function $\chi(X) \in C^{\infty}\left(\mathbb{R}_{X}^{n}\right)$ satisfying, with $r_{2}=\min \left\{r_{0}, r_{1} / 2\right\}$,

$$
\chi(X)= \begin{cases}0 & |X| \geq r_{2}, \\ 1 & |X| \leq \frac{r_{2}}{2},\end{cases}
$$

we obtain

$$
\begin{gathered}
Q_{1} \chi(X) w_{1}=-\left(1-|T|^{2}\right)^{2} \chi(X) w_{2}+f(X, T) \\
Q_{2} \chi(X) w_{2}=C_{1}\left(X, T, \partial_{X}, \partial_{T}\right) \chi(X) w_{2}+C_{2}\left(X, T, \partial_{X}, \partial_{T}\right) \chi(X) w_{1}+g(X, T)
\end{gathered}
$$

where $f(X, T)=0$ and $g(X, T)=0$ if $|X| \leq \frac{r_{2}}{2}$.

Then we see from (2.11) that

$$
\begin{aligned}
\gamma^{-1}\left(\sum_{k+|\alpha|+|\beta|=2} \gamma^{2 k}\left\|\Phi^{-\gamma}|\log r|^{-1} r^{|\alpha|+|\beta|}\left(1-|T|^{2}\right)^{|\beta|} \partial_{X}^{\alpha} \partial_{T}^{\beta} \chi(X) w_{1}\right\|^{2}\right) \\
\leq 2 C\left(\left\|r^{2} \Phi^{-\gamma} \chi(X) w_{2}\right\|^{2}+\left\|r^{2} \Phi^{-\gamma} f(X, T)\right\|^{2}\right)
\end{aligned}
$$

and

$$
\begin{aligned}
\gamma^{-1}\left(\sum_{k+|\alpha|+|\beta|=2}\right. & \left.\gamma^{2 k}\left\|\Phi^{-\gamma}|\log r|^{-1} r^{|\alpha|+|\beta|}\left(1-|T|^{2}\right)^{|\beta|} \partial_{X}^{\alpha} \partial_{T}^{\beta} \chi(X) w_{2}\right\|^{2}\right) \\
\leq & C\left(\sum_{|\alpha|+|\beta| \leq 1}\left\|\Phi^{-\gamma} r^{2}\left(1-|T|^{2}\right)^{|\beta|} \partial_{X}^{\alpha} \partial_{T}^{\beta} \chi(X) w_{2}\right\|^{2}\right. \\
& +\sum_{|\alpha|+|\beta| \leq 2}\left\|\Phi^{-\gamma} r^{2}\left(1-|T|^{2}\right)^{|\beta|} \partial_{X}^{\alpha} \partial_{T}^{\beta} \chi(X) w_{1}\right\|^{2} \\
& \left.+\left\|r^{2} \Phi^{-\gamma} g(X, T)\right\|^{2}\right),
\end{aligned}
$$

from which we draw

$$
\begin{aligned}
\gamma^{5}\left\|\Phi^{-\gamma}|\log r|^{-1} \chi(X) w_{1}\right\|^{2}+\gamma^{3} \| & \Phi^{-\gamma}|\log r|^{-1} \chi(X) w_{2} \|^{2} \\
& \leq C\left(\gamma^{2}\left\|r^{2} \Phi^{-\gamma} f(X, T)\right\|^{2}+\left\|r^{2} \Phi^{-\gamma} g(X, T)\right\|^{2}\right)
\end{aligned}
$$

for $\gamma>\gamma_{1}$ with some $\gamma_{1}>0$. Then we obtain

$$
w_{1}(X, T)=0 \text { and } w_{2}(X, T)=0 \text { for }|X| \leq \frac{r_{2}}{3} .
$$

Then $w_{1}(X, 0)=0$ for $|X| \leq \frac{r_{2}}{3}$. Since, from 2.7) and 2.8) we get $U(X, 0)=$ $W_{1}(X, 0)=w_{1}(X, 0)$, then we see that $U(x, 0)=0$ for $|x| \leq \frac{r_{2}}{3}$. Hence from (2.3) and (2.4),

$$
\int_{\mathbb{R}} u(x, t) \phi(t) d t=0 \text { for }|x| \leq \frac{r_{2}}{3} .
$$


Since (2.12) is valid for any $\psi(t) \in \gamma_{0}^{(\alpha)}((-\delta, \delta))$, then we get

$$
u(x, t)=0 \text { for }|x| \leq \frac{r_{2}}{3} \text { and }|t|<\delta .
$$

Then the proof of Theorem 1.1 is completed.

Remark 2.1. We note that the arguments above can be applied to the heat and Schrödinger coupled system

$$
\begin{aligned}
\rho_{1}(x) \partial_{t} u-\Delta_{x} u & =a_{1}\left(x, \partial_{x}\right) u+a_{2}\left(x, \partial_{x}\right) v, \\
i \rho_{2}(x) \partial_{t} v-\Delta_{x} v & =b_{1}\left(x, \partial_{x}\right) u+b_{2}\left(x, \partial_{x}\right) v
\end{aligned}
$$

with some positive functions $\rho_{j}(x)(j=1,2)$ and first-order differential operators $a_{j}\left(x, \partial_{x}\right)$ and $b_{j}\left(x, \partial_{x}\right)(j=1,2)$.

\section{APPENDIX}

In this appendix, we sketch the proof of the Carleman estimates following Alinhac and Lerner [1] (see also Tataru 7], Ch. 3, Sec. 6).

For a nonnegative function $p(x)$, when a function $a(x, t) \in C^{\infty}(U)$ satisfies, for any $\alpha$ and $\beta$,

$$
|x|^{|\alpha|}\left|\partial_{x}^{\alpha} \partial_{t}^{\beta} a(x, t)\right| \leq C_{\alpha, \beta} p(x) \text { on } U,
$$

we say that $a(x, t) \in p(x) \mathcal{O}$, where $U$ is an open subset of $\mathbb{R}_{x}^{n} \backslash\{0\} \times \mathbb{R}_{t}^{l}$.

Recall that $\Delta_{x}$ is the laplacian $\sum_{1 \leq j \leq n} \partial_{x_{j}}^{2}$. Let $\Sigma$ be an elliptic operator given by

$$
\Sigma=\sum_{1 \leq j, k \leq l} \partial_{t_{j}}\left(1-|t|^{2}\right)^{2} a_{j, k}(t) \partial_{t_{k}}
$$

where the coefficients $a_{j, k}(t)$ belonging to $C^{\infty}(V)$ with some neighborhood $V$ of $\left\{t \in \mathbb{R}^{l}|| t \mid \leq 1\right\}$ satisfy $a_{j, k}(t)=a_{k, j}(t)$ and

$$
\sum_{1 \leq j, k \leq l} \Re a_{j, k}(t) \eta_{j} \eta_{k} \geq C_{0}|\eta|^{2} \quad \text { for any } \eta \in \mathbb{R}^{l} \text { and any } t \in V
$$

with some positive constant $C_{0}$.

Let $\Phi(r)$ and $\mu$ be

$$
\begin{gathered}
\Phi(r)=(-\log r)^{\theta} r, \\
\mu=\frac{r \Phi^{\prime}(r)}{\Phi(r)}=1+\theta(\log r)^{-1}
\end{gathered}
$$

where $r=|x|$ and $\theta$ is a positive constant that will be determined later. We note that when $0<r_{0}<1$ and $0<\theta \leq\left|\log r_{0}\right| / 2$ we see that for $r \in\left(0, r_{0}\right]$,

$$
\frac{1}{2} \leq \mu<1 \text {. }
$$

Since

$$
\Phi(r)^{-\gamma} \frac{\partial}{\partial x_{k}} \Phi(r)^{\gamma}=\frac{\partial}{\partial x_{k}}+\gamma \frac{\mu}{r} \frac{x_{k}}{r},
$$

we see that for any $\gamma>0$,

$$
\Phi(r)^{-\gamma} \Delta_{x} \Phi(r)^{\gamma}=\Delta_{x}+\gamma^{2}{\frac{\mu}{r^{2}}}^{2}+\gamma\left(\frac{\mu}{r} \frac{\partial}{\partial r}+\frac{\partial}{\partial r} \frac{\mu}{r}+\frac{(n-1) \mu}{r^{2}}\right) .
$$


Let $B\left(x, t, \partial_{x}, \partial_{t}\right)$ be a second-order operator with $C^{\infty}$-coefficients vanishing when $x=0$ :

$$
B\left(x, t, x, \partial_{x}, \partial_{t}\right)=\sum_{|\alpha|+|\beta|=2} b_{\alpha, \beta}(x, t)\left(1-|t|^{2}\right)^{|\beta|} \partial_{x}^{\alpha} \partial_{t}^{\beta}
$$

where $b_{\alpha, \beta}(0, t)=0$. Then we see that the operator $B\left(x, t, \gamma, \partial_{x}, \partial_{t}\right)$ given by

$$
B\left(x, t, \gamma, \partial_{x}, \partial_{t}\right)=\Phi(r)^{-\gamma} B\left(x, t, \partial_{x}, \partial_{t}\right) \Phi(r)^{\gamma}
$$

can be written in the following way:

$$
B\left(x, t, \gamma, \partial_{x}, \partial_{t}\right)=\sum_{|\alpha|+|\beta|+k \leq 2} b_{\alpha, \beta, k}(x, t)\left(1-|t|^{2}\right)^{|\beta|} \gamma^{k} \partial_{x}^{\alpha} \partial_{t}^{\beta}
$$

where $b_{\alpha, \beta, k}(x, t) \in r^{|\alpha|+|\beta|-1} \mathcal{O}$.

Let $L$ be an operator given by

$$
L=\Delta_{x}+\Sigma+B\left(x, t, \partial_{x}, \partial_{t}\right) .
$$

We denote by $L_{\gamma}, L_{\gamma}{ }^{s}$ and $L_{\gamma}{ }^{a}$ the operators defined by

$$
L_{\gamma}=\Phi(r)^{-\gamma} L \Phi(r)^{\gamma}, L_{\gamma}{ }^{s}=\frac{1}{2}\left(L_{\gamma}+L_{\gamma}^{*}\right) \text { and } L_{\gamma}{ }^{a}=\frac{1}{2}\left(L_{\gamma}-L_{\gamma}^{*}\right)
$$

where $L_{\gamma}^{*}$ is a formal adjoint of $L_{\gamma}$, that is to say, $L_{\gamma}{ }^{s}$ and $L_{\gamma}{ }^{a}$ are the symmetric and skew-symmetric parts of $L_{\gamma}$, respectively.

Therefore we see that

$$
\begin{aligned}
& L_{\gamma}{ }^{s}=\Delta_{x}+\gamma^{2}{\frac{\mu}{r^{2}}}^{2}+\Sigma^{s}+B^{s}\left(x, t, \gamma, \partial_{x}, \partial_{t}\right), \\
& L_{\gamma}{ }^{a}=\gamma\left(\frac{\mu}{r} \frac{\partial}{\partial r}+\frac{\partial}{\partial r} \frac{\mu}{r}+\frac{(n-1) \mu}{r^{2}}\right)+\Sigma^{a}+B^{a}\left(x, t, \gamma, \partial_{x}, \partial_{t}\right),
\end{aligned}
$$

where $\Sigma^{s}$ and $\Sigma^{a}$ are the symmetric and skew-symmetric parts of $\Sigma$, respectively, and similar notation is used for $B\left(x, t, \gamma, \partial_{x}, \partial_{t}\right)$.

Then we see that for any $u(x, t)$ that is supported in $\left\{(x, t) \in \mathbb{R}_{x}^{n} \times \mathbb{R}_{t}^{l}|| x \mid \leq\right.$ $\frac{1}{2}$ and $\left.|t| \leq 1\right\}$ and flat on the plane $x=0$,

$$
\begin{aligned}
\left\|r \mu^{-\frac{1}{2}} L_{\gamma}\left(r \mu^{-\frac{1}{2}} u\right)\right\|^{2} & =\left\|r \mu^{-\frac{1}{2}} L_{\gamma}{ }^{s}\left(r \mu^{-\frac{1}{2}} u\right)\right\|^{2} \\
& +\left\|r \mu^{-\frac{1}{2}} L_{\gamma}{ }^{a}\left(r \mu^{-\frac{1}{2}} u\right)\right\|^{2}+\left(\left[r \mu^{-\frac{1}{2}} L_{\gamma}{ }^{s} r \mu^{-\frac{1}{2}}, r \mu^{-\frac{1}{2}} L_{\gamma}{ }^{a} r \mu^{-\frac{1}{2}}\right] u, u\right) .
\end{aligned}
$$

We note that

$$
\begin{gathered}
r \mu^{-\frac{1}{2}}\left(\frac{\mu}{r} \frac{\partial}{\partial r}+\frac{\partial}{\partial r} \frac{\mu}{r}+\frac{(n-1) \mu}{r^{2}}\right) r \mu^{-\frac{1}{2}}=2 r \frac{\partial}{\partial r}+n \\
{\left[r \Delta_{x} r+\gamma^{2} \mu^{2}, 2 r \frac{\partial}{\partial r}+n\right]=-4 \gamma^{2} r \mu \mu^{\prime}}
\end{gathered}
$$

and, since $\left[\Sigma^{s}, r\right]=0$,

$$
\left[r \Sigma^{s} r, 2 r \frac{\partial}{\partial r}+n\right]=-4 r^{2} \Sigma^{s}
$$

Therefore, noting that $\left[\mu^{-1 / 2}, 2 r \frac{\partial}{\partial r}+n\right]=\frac{r \mu^{\prime}}{\mu} \mu^{-1 / 2}$, we see that $\left[r \mu^{-\frac{1}{2}} L_{\gamma}{ }^{s} r \mu^{-\frac{1}{2}}\right.$, $\left.\gamma\left(2 r \frac{\partial}{\partial r}+n\right)\right]$ is equal to

$$
\gamma \frac{r \mu^{\prime}}{\mu} r \mu^{-1 / 2} L_{\gamma}{ }^{s} r \mu^{-1 / 2}+\gamma r \mu^{-1 / 2} L_{\gamma}{ }^{s} r \mu^{-1 / 2} \frac{r \mu^{\prime}}{\mu}-4 \gamma^{3} r \mu^{\prime}-4 \gamma r^{2} \mu^{-1} \Sigma^{s}+\tilde{B}^{s},
$$


where

$$
\begin{aligned}
\tilde{B}^{s} & =\mu^{-\frac{1}{2}}\left[r B^{s}\left(x, t, \gamma, \partial_{x}, \partial_{t}\right) r, \gamma\left(2 r \frac{\partial}{\partial r}+n\right)\right] \mu^{-\frac{1}{2}} \\
& =\sum_{|\alpha|+|\beta|+k \leq 2} b^{s}{ }_{\alpha, \beta, k}(x, t)\left(1-|t|^{2}\right)^{|\beta|} \gamma^{k+1} \partial_{x}^{\alpha} \partial_{t}^{\beta}
\end{aligned}
$$

with $b^{s}{ }_{\alpha, \beta, k}(x, t) \in r^{1+|\beta|+|\alpha|} \mathcal{O}$.

Hence

$$
\begin{gathered}
\left\|r \mu^{-\frac{1}{2}} L_{\gamma}{ }^{s}\left(r \mu^{-\frac{1}{2}} u\right)\right\|^{2}+\left(\left[r \mu^{-\frac{1}{2}} L_{\gamma}^{s} r \mu^{-\frac{1}{2}}, \gamma\left(2 r \frac{\partial}{\partial r}+n\right)\right] u, u\right) \\
=\left\|r \mu^{-\frac{1}{2}} L_{\gamma}^{s}\left(r \mu^{-\frac{1}{2}} u\right)+\gamma \frac{r \mu^{\prime}}{\mu} u\right\|^{2} \\
+\left(\left(-4 \gamma^{3} r \mu^{\prime}-\left(\gamma \frac{r \mu^{\prime}}{\mu}\right)^{2}\right) u, u\right)+\left(-4 \gamma r^{2} \mu^{-1} \Sigma^{s} u, u\right)+\left(\tilde{B}^{s} u, u\right) .
\end{gathered}
$$

Since $r \mu^{\prime}=-\theta(\log r)^{-2}$, we see from (3.3) that when $\theta$ is in $\left(0,\left|\log r_{0}\right| / 2\right)$ with $r_{0} \in(0,1)$, there exists a constant $\gamma_{1}$, which may depend on $r_{0}$, such that for $\gamma \geq \gamma_{1}$ we have

$$
\left(\left(-4 \gamma^{3} r \mu^{\prime}-\left(\gamma \frac{r \mu^{\prime}}{\mu}\right)^{2}\right) u, u\right) \geq 2 \gamma^{3} \theta\left\|(\log r)^{-1} u\right\|^{2}
$$

for any $u(x, t)$ that vanishes for $|x|>r_{0}$. On the other hand, from (3.1) and (3.3) we see that there exist constants $C_{1}$ and $C_{2}$ such that for any $u(x, t)$ that vanishes when $|x|>r_{0}$ or $|t|>1$, if $\theta$ is in $\left(0,\left|\log r_{0}\right| / 2\right)$ with $r_{0} \in(0,1)$, we obtain

$$
\left(-4 \gamma r^{2} \mu^{-1} \Sigma^{s} u, u\right) \geq \gamma\left(C_{1}\left\|r\left(1-|t|^{2}\right) \nabla_{t} u\right\|^{2}-C_{2}\|r u\|^{2}\right) .
$$

Since we obtain

$$
\left|\left(\tilde{B}^{s} u, u\right)\right| \leq C_{3} \gamma\left(\sum_{k+|\alpha|+|\beta| \leq 1} \gamma^{k}\left\|r^{|\alpha|+|\beta|+\frac{1}{2}}\left(1-|t|^{2}\right)^{|\beta|} \partial_{x}^{\alpha} \partial_{t}^{\beta} u\right\|\right)^{2}
$$

from (3.7), we see from (3.8), 3.9) and (3.10) that

$$
\begin{aligned}
&\left\|r \mu^{-\frac{1}{2}} L_{\gamma}{ }^{s}\left(r \mu^{-\frac{1}{2}} u\right)\right\|^{2}+\left(\left[r \mu^{-\frac{1}{2}} L_{\gamma}{ }^{s} r \mu^{-\frac{1}{2}}, \gamma\left(2 r \frac{\partial}{\partial r}+n\right)\right] u, u\right) \\
& \geq\left\|r \mu^{-\frac{1}{2}} L_{\gamma}^{s}\left(r \mu^{-\frac{1}{2}} u\right)+\gamma \frac{r \mu^{\prime}}{\mu} u\right\|^{2} \\
&+2 \gamma^{3} \theta\left\|(\log r)^{-1} u\right\|^{2}+\gamma\left(C_{1}\left\|r\left(1-|t|^{2}\right) \nabla_{t} u\right\|^{2}-C_{2}\|r u\|^{2}\right) \\
& \\
& \quad-C_{3} \gamma\left(\sum_{k+|\alpha|+|\beta| \leq 1} \gamma^{k}\left\|r^{|\alpha|+|\beta|+\frac{1}{2}}\left(1-|t|^{2}\right)^{|\beta|} \partial_{x}^{\alpha} \partial_{t}^{\beta} u\right\|\right)^{2} .
\end{aligned}
$$

Now we consider the term $\left[r \mu^{-\frac{1}{2}} L_{\gamma}{ }^{s} r \mu^{-\frac{1}{2}}, r \mu^{-\frac{1}{2}} \Sigma^{a} r \mu^{-\frac{1}{2}}\right]$. First note that

$$
\left[r \mu^{-\frac{1}{2}} \Delta_{x} r \mu^{-\frac{1}{2}}, r \mu^{-\frac{1}{2}} \Sigma^{a} r \mu^{-\frac{1}{2}}\right]=\left(\left(2 r \frac{\partial}{\partial r}+n\right)\left(\frac{2}{\mu}-\frac{r \mu^{\prime}}{\mu^{2}}\right)+b(r)\right) r^{2} \mu^{-1} \Sigma^{a}
$$

where $b(r) \in 1 \mathcal{O}$. Noting that

$$
r \mu^{-\frac{1}{2}} L_{\gamma}^{a} r \mu^{-\frac{1}{2}}=\gamma\left(2 r \frac{\partial}{\partial r}+n\right)+r^{2} \mu^{-1} \Sigma^{a}+\tilde{B}^{a},
$$


where

$$
\tilde{B}^{a}=\sum_{|\alpha|+|\beta|+k \leq 2} b^{a}{ }_{\alpha, \beta, k}(x, t)\left(1-|t|^{2}\right)^{|\beta|} \gamma^{k} \partial_{x}^{\alpha} \partial_{t}^{\beta}
$$

with $b^{a}{ }_{\alpha, \beta, k}(x, t) \in r^{1+|\alpha|+|\beta|} \mathcal{O}$, we see that

$$
\begin{aligned}
& \left\|r \mu^{-\frac{1}{2}} L_{\gamma}{ }^{a}\left(r \mu^{-\frac{1}{2}} u\right)\right\|^{2} \\
& +\Re\left(\left(2 r \frac{\partial}{\partial r}+n\right)\left(\frac{2}{\mu}-\frac{r \mu^{\prime}}{\mu^{2}}\right) r^{2} \mu^{-1} \Sigma^{a} u, u\right) \\
& =\left\|\left(\gamma\left(2 r \frac{\partial}{\partial r}+n\right)+\left(1-\frac{1}{2 \gamma}\left(\frac{2}{\mu}-\frac{r \mu^{\prime}}{\mu^{2}}\right)\right)\left(r^{2} \mu^{-1} \Sigma^{a}+\tilde{B}^{a}\right)\right) u\right\|^{2} \\
& \quad-\Re\left(\left(2 r \frac{\partial}{\partial r}+n\right)\left(\frac{2}{\mu}-\frac{r \mu^{\prime}}{\mu^{2}}\right) \tilde{B}^{a} u, u\right) \\
& +\left\|\left(r^{2} \mu^{-1} \Sigma^{a}+\tilde{B}^{a}\right) u\right\|^{2}-\left\|\left(1-\frac{1}{2 \gamma}\left(\frac{2}{\mu}-\frac{r \mu^{\prime}}{\mu^{2}}\right)\right)\left(r^{2} \mu^{-1} \Sigma^{a}+\tilde{B}^{a}\right) u\right\|^{2}
\end{aligned}
$$

Since for $r \in\left(0, r_{0}\right], \theta \in\left(0,\left|\log r_{0}\right| / 2\right)$ and $\gamma \geq \gamma_{2}$ with some $\gamma_{2}>0$ we have $0<\frac{1}{2 \gamma}\left(\frac{2}{\mu}-\frac{r \mu^{\prime}}{\mu^{2}}\right)<1$, we see from (3.12) that the right-hand side of (3.13) is not smaller than

$$
-C\left(\sum_{|\alpha| \leq 1}\left\|r^{|\alpha|+\frac{1}{2}} \partial_{x}^{\alpha} u\right\|\right)\left(\sum_{k+|\alpha|+|\beta| \leq 2} \gamma^{k}\left\|r^{|\alpha|+|\beta|+\frac{1}{2}}\left(1-|t|^{2}\right)^{|\beta|} \partial_{x}^{\alpha} \partial_{t}^{\beta} u\right\|\right) .
$$

Since $b(r)$ is real-valued and $\Sigma^{a}$ is skew-symmetric, we see from $\left[r, \Sigma^{a}\right]=0$ that $\Re\left(b(r) r^{2} \mu^{-1} \Sigma^{a} u, u\right)=0$. Then the left-hand side of (3.13) is equal to

$$
\left\|r \mu^{-\frac{1}{2}} L_{\gamma}^{a}\left(r \mu^{-\frac{1}{2}} u\right)\right\|^{2}+\left(\left[r \mu^{-\frac{1}{2}} \Delta_{x} r \mu^{-\frac{1}{2}}, r \mu^{-\frac{1}{2}} \Sigma^{a} r \mu^{-\frac{1}{2}}\right] u, u\right) .
$$

Since we see from (3.4) that

$$
\begin{aligned}
&\left|\left(\left[r^{2} \mu^{-1} \Sigma^{s}, r^{2} \mu^{-1} \Sigma^{a}\right] u, u\right)\right|+\left|\left(\left[r \mu^{-\frac{1}{2}} B^{s} r \mu^{-\frac{1}{2}}, r^{2} \mu^{-1} \Sigma^{a}\right] u, u\right)\right| \\
& \leq C\left(\sum_{|\beta| \leq 1}\left\|r^{|\beta|+\frac{1}{2}}\left(1-|t|^{2}\right)^{|\beta|} \partial_{t}^{\beta} u\right\|\right) \\
& \times\left(\sum_{k+|\alpha|+|\beta| \leq 2} \gamma^{k}\left\|r^{|\alpha|+|\beta|+\frac{1}{2}}\left(1-|t|^{2}\right)^{|\beta|} \partial_{x}^{\alpha} \partial_{t}^{\beta} u\right\|\right),
\end{aligned}
$$

we obtain the following:

$$
\begin{gathered}
\left\|r \mu^{-\frac{1}{2}} L_{\gamma}{ }^{a}\left(r \mu^{-\frac{1}{2}} u\right)\right\|^{2}+\left(\left[r \mu^{-\frac{1}{2}} L_{\gamma}{ }^{s} r \mu^{-\frac{1}{2}}, r \mu^{-\frac{1}{2}} \Sigma^{a} r \mu^{-\frac{1}{2}}\right] u, u\right) \\
\geq-C\left(\sum_{|\alpha|+|\beta| \leq 1}\left\|r^{|\alpha|+|\beta|+\frac{1}{2}}\left(1-|t|^{2}\right)^{|\beta|} \partial_{x}^{\alpha} \partial_{t}^{\beta} u\right\|\right) \\
\times\left(\sum_{k+|\alpha|+|\beta| \leq 2} \gamma^{k}\left\|r^{|\alpha|+|\beta|+\frac{1}{2}}\left(1-|t|^{2}\right)^{|\beta|} \partial_{x}^{\alpha} \partial_{t}^{\beta} u\right\|\right) .
\end{gathered}
$$


Finally from (3.4) we see that

$$
\begin{aligned}
\left|\left(\left[r \mu^{-\frac{1}{2}} L_{\gamma}{ }^{s} r \mu^{-\frac{1}{2}}, r \mu^{-\frac{1}{2}} B^{a} r \mu^{-\frac{1}{2}}\right] u, u\right)\right| & \\
\leq C\left(\sum_{|\alpha|+|\beta|+k \leq 1} \gamma^{k}\left\|r^{|\alpha|+|\beta|+\frac{1}{2}}\left(1-|t|^{2}\right)^{|\beta|} \partial_{x}^{\alpha} \partial_{t}^{\beta} u\right\|\right) & \\
& \times\left(\sum_{k+|\alpha|+|\beta| \leq 2} \gamma^{k}\left\|r^{|\alpha|+|\beta|+\frac{1}{2}}\left(1-|t|^{2}\right)^{|\beta|} \partial_{x}^{\alpha} \partial_{t}^{\beta} u\right\|\right) .
\end{aligned}
$$

From (3.6) and the ellipticity (3.1), we obtain

$$
\begin{aligned}
\|(\log r)^{-1}\left(r \mu^{-\frac{1}{2}} L_{\gamma}{ }^{s}\right. & \left.\left(r \mu^{-\frac{1}{2}} u\right)+\gamma \frac{r \mu^{\prime}}{\mu} u\right)\left\|^{2}+C_{1} \gamma^{4}\right\|(\log r)^{-1} u \|^{2} \\
& \geq C_{0} \sum_{|\alpha|+|\beta|+k=2}\left(\gamma^{k}\left\|(\log r)^{-1} r^{|\alpha|+|\beta|}\left(1-|t|^{2}\right)^{|\beta|} \partial_{x}^{\alpha} \partial_{t}^{\beta} u\right\|\right)^{2}
\end{aligned}
$$

for $\gamma>\gamma_{3}$ with some $\gamma_{3}>0$ and some positive constants $C_{0}$ and $C_{1}$. Therefore by taking a small enough $r_{0}>0$ and picking $\theta=1$, we see that for any $u(x, t)$ supported in $\left\{(x, t) \in \mathbb{R}_{x}^{n} \times \mathbb{R}_{t}^{l}|| x \mid \leq r_{0}\right.$ and $\left.|t| \leq 1\right\}$ and flat on $x=0$,

$$
\begin{aligned}
\left\|r \mu^{-\frac{1}{2}} L_{\gamma}\left(r \mu^{-\frac{1}{2}} u\right)\right\|^{2} & \\
& \geq C_{0} \sum_{|\alpha|+|\beta|+k=2} \gamma^{-1}\left(\gamma^{k}\left\|(\log r)^{-1} r^{|\alpha|+|\beta|}\left(1-|t|^{2}\right)^{|\beta|} \partial_{x}^{\alpha} \partial_{t}^{\beta} u\right\|\right)^{2}
\end{aligned}
$$

for $\gamma>\gamma_{0}$ with some positive constants $\gamma_{0}$ and $C_{0}$. Then from (3.5) we obtain the following Carleman estimates.

Lemma 3.1 (Alinhac-Lerner [1]). There exist positive constants $r_{0}, \gamma_{0}$ and $C_{0}$ such that for any $u(x, t)$ supported in $\left\{(x, t) \in \mathbb{R}_{x}^{n} \times \mathbb{R}_{t}^{l}|| x \mid \leq r_{0}\right.$ and $\left.|t| \leq 1\right\}$ and flat on $x=0$,

$$
\begin{aligned}
& \left\|\Phi(r)^{-\gamma} r^{2} L u\right\|^{2} \\
& \quad \geq C_{0} \sum_{|\alpha|+|\beta|+k=2} \gamma^{-1}\left(\gamma^{k}\left\|\Phi(r)^{-\gamma}(\log r)^{-1} r^{|\alpha|+|\beta|}\left(1-|t|^{2}\right)^{|\beta|} \partial_{x}^{\alpha} \partial_{t}^{\beta} u\right\|\right)^{2}
\end{aligned}
$$

for $\gamma>\gamma_{0}$ where $\Phi(r)$ is given by (B.2) with $\theta=1$.

\section{REFERENCES}

[1] S. Alinhac and N. Lerner, Unicité forte à partir d'une variété de dimension quelconque pour des inégalités différentielles elliptiques. Duke Math. J. 48 (1981), no. 1, 49-68. MR 83b:35005a

[2] F. Colombini and C. Grammatico, Strong uniqueness for Laplace and bi-Laplace operators in the limit case. Carleman estimates and applications to uniqueness and control theory (Cortona, 1999), 49-60, Progr. Nonlinear Differential Equations Appl., 46, Birkhäuser Boston, Boston, MA, 2001. MR 2003e:35333

[3] V. Isakov, On uniqueness in a lateral Cauchy problem with multiple characteristics, J. Differential Equations 134 (1997), 134-147 MR 97m:35005

[4] G. Lebeau, Un problème d'unicité forte pour l'équation des ondes. Comm. Partial Differential Equations 24 (1999), no. 3-4, 777-783 MR 2000c:58054

[5] L. Robbiano, Théorème d'unicité adapté au contrôle des solutions des problèmes hyperboliques. Comm. Partial Differential Equations 16 (1991), no. 4-5, 789-800. MR 92j:35002 
[6] D. Tataru, Unique continuation for solutions to PDE's: between Hörmander's theorem and Holmgren's theorem, Comm. Partial Differential Equations 20 (1995), 855-884. MR 96e:35019

[7] D. Tataru, Carleman estimates, unique continuation and applications, (http://www.math. berkeley.edu/ tataru/papers/)

Laboratory of Applied Mathematics, Graduate School of Engineering, Osaka City UNIVERSITY, OSAKA, 558-8585, JAPAN

E-mail address: starama@mech.eng.osaka-cu.ac.jp 